\title{
A STRONG DUALITY THEOREM FOR SEPARABLE LOCALLY COMPACT GROUPS
}

\author{
BY \\ JOHN ERNEST
}

\begin{abstract}
We obtain a duality theorem for separable locally compact groups, where the group is regained from the set of factor unitary representations. Loosely stated, the group is isomorphic to the group of nonzero bounded, operator valued maps on the set of factor representations, which preserve unitary equivalence, direct sums, and tensor products. The axiom involving tensor products is formulated in terms of direct integral theory. The topology of $G$ may be regained from the irreducible representations alone. Indeed a sequence $\left\{x_{i}\right\}$ in $G$, converges to $x$ in $G$ if and and only if $\pi\left(x_{i}\right)$ converges strongly to $\pi(x)$ for each irreducible representation $\pi$ of $G$. This result supplies the missing topological part of the strong duality theorem of N. Tatsuuma for type I separable locally compact groups (based on irreducible representations). Our result also generalizes this Tatsuuma strong duality theorem to the nontype I case.
\end{abstract}

1. Introduction. N. Tatsuuma has obtained a remarkable duality theorem for locally compact groups [10, Theorem 1], where a locally compact group is reconstructed from its infinite-dimensional unitary representation theory, in a manner completely analogous to the way the Tannaka duality theory reconstructs a compact group from its finite-dimensional representations. However the Tatsuuma theorem reconstructs the group from the set of general unitary representations (particularly the left regular representation), while both the Tannaka duality theorem for compact groups and the Pontrjagen duality theorem for abelian locally compact groups reconstructs the group from the set of irreducible unitary representations. Unfortunately, for a general locally compact group, a unitary representation need not be a direct sum of irreducible representations. Further in any such duality theory the tensor product operation is crucial for the structure of the representation theory, and the tensor product of two irreducible representations is, in general, not a direct sum of irreducible representations. Thus to obtain a duality theorem based on the space of irreducible representations, the theory of direct integrals of representations must be used to even specify the tensor product structure. Thus we must restrict ourselves to separable (second countable) locally compact groups and to separable unitary representations in order that the direct integral theory (cf. [2], [3], [6] and [7]) applies. Further this direct integral theory

Received by the editors November 24, 1969.

AMS 1969 subject classifications. Primary 2220, 2260; Secondary 4615, 4665.

Key words and phrases. Separable locally compact group, unitary representation, factor representation, Tatsuuma duality, Borel options.

Copyright (C) 1971, American Mathematical Society 
is much better behaved for type I groups, where every representation has an essentially unique decomposition as a direct integral of irreducible representations. N. Tatsuuma has obtained a duality theorem for type I separable locally compact groups, based on the set of irreducible representations of the group (cf. $\$ 4$ of [10]). $\mathrm{N}$. Tatsuuma refers to this result as the duality theorem in a strong form. Here the hypothesis of the theorem uses the measure or Borel structure on the set of irreducible representations, in addition to the structure involving direct sums, unitary equivalence and tensor products. However the topological portion of this type I strong duality theorem is somewhat deficient, as it is not clear how the topology of the group may be specified purely in terms of its irreducible representations. Nevertheless this is analyzed for the case of abelian and compact groups, and the complete Pontrjagin and Tannaka duality theorems are obtained as special cases of Tatsuuma's strong duality theorem (cf. $\$ 4.3$ of [10]).

In $\$ 4$ we obtain the topological portion of the theorem, even in the nontype I case. Thus in any separable locally compact group $G$ a sequence $x_{i}$ converges to an element $x$ in $G$ if and only if $\pi\left(x_{i}\right)$ converges strongly to $\pi(x)$ for every irreducible representation of $G$.

The main point of this paper is to generalize the strong form of Tatsuuma duality to the nontype I case, where the decomposition of a representation into irreducibles is no longer unique. We have formulated our main result (cf. Definitions 3.1, 3.2 and Theorem 3.3) for separable locally compact groups in a somewhat different manner than Tatsuuma's type I theorem, although it reduces to essentially the same result in the type I case. The biggest change, in going to the nontype I case, is the necessity of using the set of all factor representations rather than merely irreducible representations, as a dual object. (We admit defeat in the terminology battle and with this paper we begin using the term "factor" rather than the term "primary" employed in [3].) The concept "set $G_{f}$ of factor representations of $G$ " is made more explicit in Definition 2.1, and $G_{f}$ is given a Borel structure. Very loosely the strong duality theorem may be formulated as follows. Let $G^{+}$denote the set of maps (called options) on $G_{f}$ which associate a nonzero operator on the space of $\pi$, for each $\pi$ in $G_{f}$ and which are bounded Borel maps which preserve direct sums, unitary equivalence and tensor products. Since factor representations are not closed under tensor products, this last condition must be formulated somewhat technically in terms of direct integral theory (cf. axiom (iii) of Definition 3.1). Give $G^{+}$the largest topology such that $T_{i}$ converges to $T$ whenever $T_{i}$ is a sequence such that $T_{i}(\pi)$ converges strongly to $T(\pi)$, for all $\pi$ in $G_{f}$ (cf. Remark 4.5). For each $x$ in $G$, one may define an element $\hat{x}$ of $G^{+}$by $\hat{x}(\pi)=\pi(x)$ for all $\pi$ in $G_{f}$. The strong duality theorem (Theorem 3.3) then asserts that the map $x \rightarrow \hat{x}$ is an isomorphism and homeomorphism of $G$ onto $G^{+}$. The topological portion of this theorem is new, even in the type I case.

Our main technique is to study Borel options (bounded Borel operator valued maps on $G_{f}$ which preserve unitary equivalence and direct sums) and to show how 
these can be canonically extended by integration theory to options defined for all separable representations. In this way we reduce the strong duality theorem to the weak form of the Tatsuuma duality which involves all representations ( $\$ 2$ of [10]). This theory of Borel options is developed in $\$ 2$. While this material is formulated in terms of separable unitary representations of a separable locally compact group, it applies equally well to separable ${ }^{*}$-representations of separable $C^{*}$-algebras. We have thus written up this theory in one separate section for its potential utility in solving quite different problems. In particular the theory of $\$ 2$ might be useful for obtaining a strong form of the Takesaki duality theorem for separable $C^{*}$-algebras (cf. [9]).

Throughout this paper $G$ will always denote a separable (second countable) locally compact group, and "representation" shall always mean strongly continuous unitary representation on a separable Hilbert space.

A portion of this work was done while the author was a visitor at the Mathematisches Institut der Universität, at Göttingen, Germany, during the summer of 1969 and he wishes to express his gratefulness for the hospitality of the Institute. He also thanks the National Science Foundation, U.S.A., for its financial support.

2. Borel options. In our previous discussion of duality theory (cf. [4]) we have used a slightly different concrete dual object than that which has been used in decomposition theory ([7] and [3]). Since the major technique for obtaining the duality theorem of this paper is the direct integral decomposition theory of representations, we shall make use of this different concrete dual, which we now define. Throughout this paper, $G$ is a separable, locally compact group.

Definition 2.1. Let $\mathscr{H}_{\infty}$ denote the classical Hilbert space of square summable sequences of complex numbers. Let $\mathscr{H}_{n}$ denote the finite-dimensional subspace consisting of all sequences which are zero after the $n$th term. Let $G_{\infty}^{c}$ denote the set of all concrete unitary representations of $G$ on $\mathscr{H}_{\infty}$ and $G_{n}^{c}$ the set of all such representations on $\mathscr{H}_{n}$. Let

$$
G^{c}=\bigcup_{n=\infty, 1,2, \ldots} G_{n}^{c}
$$

The set $G^{c}$ is given the Mackey Borel structure by specifying that each $G_{n}^{c}$ is a Borel subset of $G^{c}$, and each $G_{n}^{c}$ is given the smallest Borel structure making the complex functions $\pi \rightarrow(\pi(x) \psi, \varphi)$ Borel functions, for each $x$ in $G$ and $\psi, \varphi$ in $\mathscr{H}_{n}$.

Let $G_{f}$ denote the set of all factor representations in $G^{c}$. The set $G_{f}$ is a Borel subset of $G^{c}$ and hence a standard Borel space (cf. Theorem 1 and its corollary, [3]). Finally the quasi-dual, $\tilde{G}$, is the quotient of $G_{f}$, with respect to quasi-equivalence.

This concrete dual, $G^{c}$, is precisely that defined in [7] and used in [3]. In our earlier paper on duality [4], we defined the concrete dual to be the set of all unitary representations of $G$ which act on any closed subspace of $\mathscr{H}_{\infty}$. However every representation in this larger concrete dual is unitary equivalent to one contained in $G^{c}$ and hence, by axiom [4] of Definition 2.1 of [4], every option defined on the larger 
concrete dual is uniquely determined by its values on $G^{c}$. Hence, in the case of separable locally compact groups, the entire theory of [4] applies, using this slightly smaller concrete dual $G^{c}$. In this context we next define what we mean by an option on the factor dual $G_{f}$.

Definition 2.2. An option on $G_{f}$ is an operator valued function $T$, defined on $G_{f}$, satisfying the following three axioms:

(i) $T(\pi) \in \mathscr{L}(\mathscr{H}(\pi))$ for all $\pi$ in $G_{f}$.

(ii) $\operatorname{Sup}_{\pi \in G_{f}}\|T(\pi)\|<+\infty$.

(iii) If $\pi, \pi^{\prime}$ are elements of $G_{f}$, and if $U$ is a linear isometry of $\mathscr{H}\left(\pi^{\prime}\right)$ onto a closed subspace of $\mathscr{H}(\pi)$ such that $U \pi^{\prime} U^{-1} \leqq \pi$, then $\mathscr{H}\left(U \pi^{\prime} U^{-1}\right)$ is an invariant subspace of the operator $T(\pi)$, and the restriction of $T(\pi)$ to $\mathscr{H}\left(U \pi^{\prime} U^{-1}\right)$ is precisely $U T\left(\pi^{\prime}\right) U^{-1}$.

REMARK 2.3. An option on $G^{c}$ is defined in precisely the same manner, with $G^{c}$ replacing $G_{f}$ in the above definition. In this definition, and throughout this paper, $\mathscr{H}(\pi)$ will always denote the representation space of the representation $\pi$, and $\mathscr{L}(\mathscr{H}(\pi))$ will denote the space of all bounded linear operators on $\mathscr{H}(\pi)$. Similarly, for any unitary representation $\pi, \mathscr{A}(\pi)$ will always denote the von Neumann algebra generated by the range of $\pi$. Loosely speaking, an option on $G_{f}$ is a uniformly bounded operator valued function on $G_{f}$ which preserves subrepresentations and unitary equivalence.

If $\pi$ is any factor representation on a separable (possibly finite dimensional) Hilbert space $\mathscr{H}$, then $\pi$ is unitary equivalent to an element of $G_{f}$, say $U \pi U^{-1} \in G_{f}$. If $T$ is an option on $G_{f}$, we shall define $T(\pi)=U^{-1} T\left(U \pi U^{-1}\right) U$. This convention for the automatic extension of an option on $G_{f}$ to separable factor representations not in $G_{f}$ will help us to avoid a proliferation of isometries. In terms of this convention, axiom (iii) of Definition 2.2 may be formulated as stating that if $\pi$ and $\pi^{\prime}$ are separable factor representations and $\pi^{\prime} \leqq \pi$, then $T\left(\pi^{\prime}\right)$ is the restriction of $T(\pi)$ to $\mathscr{H}\left(\pi^{\prime}\right)$.

In a previous paper [4] we have shown that the set of options on $G^{c}$ form a von Neumann algebra isomorphic to the enveloping von Neumann algebra of $G$. We next state the corresponding fact for options defined only on $G_{f}$, and leave the proof to the reader.

Proposition 2.4. The set of options on $G_{f}$, with norm defined by

$$
\|T\|=\operatorname{Sup}_{\pi \in G_{f}}\|T(\pi)\|
$$

and *-algebra operations defined pointwise, is a von Neumann algebra canonically isomorphic to the von Neumann algebra generated by the range of the representation which is the direct sum of all representations in $G_{f}$.

Proposition 2.5. If $T$ is an option on $G_{f}$, then $T(\pi) \in \mathscr{A}(\pi)$ for every $\pi$ in $G_{f}$.

Proof. Let $T$ be an option on $G_{f}$ and $\pi$ an element of $G_{f}$. Let $E$ be a projection in $\mathscr{A}(\pi)^{\prime}$, the commutant of $\mathscr{A}(\pi)$. Then the range of $E$ is an invariant subspace of $\pi$ 
and hence, by axiom (iii) of Definition 2.2, an invariant subspace of $T(\pi)$. Similarly the range of $I-E$ is an invariant subspace of $T(\pi)$. Thus $T(\pi)$ commutes with all the projections of $\mathscr{A}(\pi)^{\prime}$ and hence $T(\pi) \in \mathscr{A}(\pi)$.

Definition 2.6. An option $T$ on $G_{f}$ will be called a Borel option if the complex valued functions $\pi \rightarrow(T(\pi) \psi, \varphi)$ are Borel functions on $G_{f}^{n}=G_{f} \cap G_{n}^{c}$, for all $\psi, \varphi$ in $\mathscr{H}_{n}$ and for $n=\infty, 1,2, \ldots$

We mention some examples of Borel options. If $x$ is an element of $G$, the option $\hat{x}$, defined on $G_{f}$ by $\hat{x}(\pi)=\pi(x)$ for all $\pi$ in $G_{f}$, is a Borel option, precisely by the definition of the Borel structure on $G_{f}$. Similarly, if $g$ is an element of $C^{*}(G)$, the $C^{*}$-group algebra of $G$, then $\hat{g}$ is a Borel option on $G_{f}$, where $\hat{g}(\pi)=\pi^{\prime}(g)$, where $\pi \in G_{f}$ and $\pi^{\prime}$ denotes the corresponding (i.e., integrated) proper ${ }^{*}$-representation of $C^{*}(G)$. Further linear combinations (defined pointwise) of Borel options on $G_{f}$ are Borel options.

We next consider the problem of extending a Borel option on $G_{f}$, to all of $G^{c}$. In general, a Borel option on $G_{f}$ will admit many extensions to the entire concrete dual $G^{c}$. For example, the identity option $I$ on $G_{f}(I(\pi)$ is the identity operator on $\mathscr{H}(\pi)$ for every $\pi$ in $G_{f}$ ) is clearly a Borel option. (In fact $I=\hat{e}$, where $e$ is the identity element of the group.) Note that every $\pi$ in $G^{c}$ may be written uniquely in the form

$$
\pi=\pi_{d} \oplus \pi_{c}
$$

where $\pi_{d}$ and $\pi_{c}$ are disjoint, $\pi_{d}$ is a direct sum of factor representations, and $\pi_{c}$ contains no factor subrepresentations. For each $\pi$ in $G^{c}$, let $D(\pi)$ denote the projection of $\mathscr{H}(\pi)$ onto $\mathscr{H}\left(\pi_{d}\right)$. Then $D$ is also an option on $G^{c}$, which extends $I$. However, while every Borel option on $G_{f}$ does not admit a unique extension to $G^{c}$, we shall now see that every such Borel option admits a canonical extension to $G^{c}$. (In the case of this example, the identity option on $G^{c}$ will be the canonical extension of the identity option on $G_{f}$.)

We consider the central decomposition of an arbitrary element $\pi$ of $G^{c}$. Thus there exists a Borel subset $B \subset G$ such that $B$ is a standard Borel space, a $\sigma$-finite Borel measure $\nu$ on $\tilde{G}$ such that $\nu(\tilde{G}-B)=0$, and a Borel map $\xi \rightarrow \pi(\xi)$ of $B$ into $G_{f}$ such that $\pi(\xi) \in \xi$ for every $\xi$ in $B$. Then $\int_{B} \pi(\xi) d \nu(\xi)$ is unitary equivalent to $\pi$ and is the central decomposition of $\pi$. The range of the associated projection valued measure is precisely the set of projections in the center of $\int_{B} \pi(\xi) d \nu(\xi)$ (cf. Theorem 2 and its corollary of [3]). Of course

$$
\begin{aligned}
\mathscr{H}\left(\int_{B} \pi(\xi) d \nu(\xi)\right) & =\int_{B} \mathscr{H}(\pi(\xi)) d \nu(\xi) \\
& =\sum_{n=\infty, 1,2, \ldots} \oplus L^{2}\left(B_{n}, \mathscr{H}_{n}, \nu\right)
\end{aligned}
$$

where $B_{n}=\left\{\xi: \mathscr{H}(\pi(\xi))=\mathscr{H}_{n}\right\}, n=\infty, 1,2, \ldots$, and $L^{2}\left(B_{n}, \mathscr{H}_{n}, \nu\right)$ denotes the Hilbert space of $\nu$-square integrable functions from $B_{n}$ to $\mathscr{H}_{n}$. 
Let $V$ denote the linear isometry of $\mathscr{H}(\pi)$ onto $\int_{B} \mathscr{H}(\pi(\xi)) d \nu(\xi)$ such that

$$
V \pi V^{-1}=\int_{B} \pi(\xi) d \nu(\xi)
$$

Let $T$ denote a Borel option on $G_{f}$. By a trinary rationale, we shall use $T^{c}$ to denote the canonical extension of $T$, to the concrete dual $G^{c}$, by means of the central decomposition, defined by

$$
T^{c}(\pi)=V^{-1}\left(\int_{B} T(\pi(\xi)) d \nu(\xi)\right) V .
$$

THEOREM 2.7. If $T$ is a Borel option on $G_{f}$, then the canonical extension $T^{c}$ is a well-defined option on $G^{c}$, such that $\left\|T^{c}\right\|=\|T\|$.

Proof. Consider two possible central decompositions of $\pi$, say

$$
\pi=V_{1}^{-1} \pi_{1} V_{1} \quad \text { where } \pi_{1}=\int_{B_{1}} \pi_{1}(\xi) d \nu_{1}(\xi)
$$

and

$$
\pi=V_{2}^{-1} \pi_{2} V_{2} \quad \text { where } \pi_{2}=\int_{B_{2}} \pi_{2}(\xi) d \nu_{2}(\xi),
$$

where $\xi \rightarrow \pi_{1}(\xi)$ and $\xi \rightarrow \pi_{2}(\xi)$ are Borel fields of factor representations on $B_{1}$ and $B_{2}$ respectively. Thus $\pi_{1}(\xi) \in G_{f}$ for all $\xi \in B_{1}$ and $\pi_{2}(\xi) \in G_{f}$ for all $\xi \in B_{2}$. By the essential uniqueness of the central decomposition, we have that $\nu_{1}$ and $\nu_{2}$ are equivalent measures on $\tilde{G}$ (i.e., have the same null sets) and $B=B_{1} \cap B_{2}$ is a Borel subset of $\tilde{G}$ such that $\nu_{1}\left(B_{1}-B\right)=0$ and $\nu_{2}\left(B_{2}-B\right)=0$. Thus we may identify

$$
\int_{B_{i}} \pi_{i}(\xi) d \nu_{i}(\xi) \text { with } \int_{B} \pi_{i}(\xi) d \nu_{i}(\xi)
$$

for $i=1,2$. Thus, without loss of generality we shall take $B_{1}=B_{2}=B$ in the above decompositions. Further since $\pi_{1}(\xi) \cong \pi_{2}(\xi)$, except for a set of measure zero, we shall assume $B$ has been chosen such that $\pi_{1}(\xi) \cong \pi_{2}(\xi)$ for all $\xi$ in $B$. Since $\pi_{i}(\xi) \in G_{f}$, $i=1,2$, equality of dimension implies they are in fact acting on the same space. Thus in particular, $\mathscr{H}\left(\pi_{1}(\xi)\right)=\mathscr{H}\left(\pi_{2}(\xi)\right)$ for all $\xi$ in $B$.

Since $\nu_{1}$ and $\nu_{2}$ are equivalent measures, there exists a canonical isomorphism $V_{3}$ of $\mathscr{H}\left(\pi_{2}\right)=\int_{B} \mathscr{H}\left(\pi_{2}(\xi)\right) d \nu_{2}(\xi)$ onto $\int_{B} \mathscr{H}\left(\pi_{2}(\xi)\right) d \nu_{1}(\xi)$ (cf. remark on p. 148 of [1]). Let $\pi_{3}=\int_{B} \pi_{2}(\xi) d \nu_{1}(\xi)$. Then $\pi_{2}=V_{3}^{-1} \pi_{3} V_{3}$ and $\pi=\left(V_{3} V_{2}\right)^{-1} \pi_{3}\left(V_{3} V_{2}\right)$. Let $U=V_{3} V_{2} V_{1}^{-1}$. Then

$$
\pi_{1}=V_{1} \pi V_{1}^{-1}=V_{1} V_{2}^{-1} V_{3}^{-1} \pi_{3} V_{3} V_{2} V_{1}^{-1}=U^{-1} \pi_{3} U
$$

Since $\mathscr{H}\left(\pi_{1}(\xi)\right)=\mathscr{H}\left(\pi_{2}(\xi)\right)$ for all $\xi$ in $B$ we have $\mathscr{H}\left(\pi_{1}\right)=\mathscr{H}\left(\pi_{3}\right)$. Thus $U$ is a unitary operator on $\mathscr{H}\left(\pi_{1}\right)$ which is decomposable (cf. Proposition 8.2.4 of [2]). In fact

$$
U=\int_{B} U(\xi) d \nu_{1}(\xi)
$$


where, for $\nu_{1}$-almost all $\xi, U(\xi)$ is a unitary operator on $\mathscr{H}\left(\pi_{1}(\xi)\right)$ such that

$$
\pi_{1}(\xi)=U(\xi)^{-1} \pi_{2}(\xi) U(\xi) .
$$

Indeed, eliminating this null set by choosing a smaller Borel set $B$, if necessary, we may assume, without loss of generality, that $U(\xi)$ is a unitary operator on $\mathscr{H}\left(\pi_{1}(\xi)\right)$ such that $\pi_{1}(\xi)=U(\xi)^{-1} \pi_{2}(\xi) U(\xi)$ for all $\xi$ in $B$.

We are now in a position to see that $T^{c}$ is well defined. By axiom (iii) (Definition 2.2) for an option on $G_{f}$ we have, for each $\xi$,

Hence

$$
T\left(\pi_{1}(\xi)\right)=U(\xi)^{-1} T\left(\pi_{2}(\xi)\right) U(\xi) .
$$

$$
\begin{aligned}
V_{1}^{-1}\left(\int_{B} T\left(\pi_{1}(\xi)\right) d \nu_{1}(\xi)\right) V_{1} & =V_{1}^{-1}\left(\int_{B} U(\xi)^{-1} T\left(\pi_{2}(\xi)\right) U(\xi) d \nu_{1}(\xi)\right) V_{1} \\
& =V_{1}^{-1} U^{-1}\left(\int_{B} T\left(\pi_{2}(\xi)\right) d \nu_{1}(\xi)\right) U V_{1} \\
& =V_{1}^{-1} U^{-1} V_{3}\left(\int_{B} T\left(\pi_{2}(\xi)\right) d \nu_{2}(\xi)\right) V_{3}^{-1} U V_{1} \\
& =V_{2}^{-1}\left(\int_{B} T\left(\pi_{2}(\xi)\right) d \nu_{2}(\xi)\right) V_{2}
\end{aligned}
$$

Hence $T^{c}(\pi)$ is well defined.

As a corollary of this verification, we observe that $T^{c}$ satisfies a part of axiom (iii) (cf. Definition 2.2) of an option on $G^{c}$. Specifically, if $\pi_{1}$ and $\pi_{2}$ are in $G^{c}$ and $U$ is a linear isometry of $\mathscr{H}\left(\pi_{1}\right)$ onto $\mathscr{H}\left(\pi_{2}\right)$ such that $\pi_{1}=U^{-1} \pi_{2} U$, then $T^{c}\left(\pi_{1}\right)$ $=U^{-1} T^{c}\left(\pi_{2}\right) U$. Indeed if $\pi_{2}$ has a central decomposition

$$
\pi_{2}=V^{-1}\left(\int_{B} \pi(\xi) d \nu(\xi)\right) V
$$

then $\pi_{1}$ has a central decomposition

and hence

$$
\pi_{1}=U^{-1} V^{-1}\left(\int_{B} \pi(\xi) d \nu(\xi)\right) V U
$$

$$
T^{c}\left(\pi_{1}\right)=U^{-1} V^{-1} \int_{B} T(\pi(\xi)) d \nu(\xi) V U=U^{-1} T^{c}\left(\pi_{2}\right) U
$$

The map $T^{c}$ on $G^{c}$ clearly satisfies axiom (i) of Definition 2.2. We next show that $T^{c}$ is uniformly bounded on $G^{c}$ (axiom (ii) of Definition 2.2).

If $\pi \in G^{c}$, then $\pi$ admits a central decomposition $\pi=V^{-1} \int_{B} \pi(\xi) d \nu(\xi) V$. Hence

$$
\begin{aligned}
\left\|T^{c}(\pi)\right\| & =\left\|\int_{B} T(\pi(\xi)) d \nu(\xi)\right\| \\
& =\nu \text {-ess. } \sup \|T(\pi(\xi))\| \\
& \leqq \operatorname{Sup}_{\pi^{\prime} \in G_{f}}\left\|T\left(\pi^{\prime}\right)\right\| .
\end{aligned}
$$


Hence

$$
\left\|T^{c}\right\|=\operatorname{Sup}_{\pi \in G^{c}}\left\|T^{c}(\pi)\right\| \leqq \operatorname{Sup}_{\pi \in G_{f}}\|T(\pi)\|=\|T\| .
$$

The reverse inequality is trivial since $G_{f} \subset G^{c}$. Hence $\left\|T^{c}\right\|=\|T\|$ and we have established everything in our theorem, except the fact that $T^{c}$ satisfies axiom (iii) of Definition 2.2.

Suppose $\pi, \pi^{\prime}$ are elements of $G^{c}$ and $U$ is a linear isometry of $\mathscr{H}\left(\pi^{\prime}\right)$ onto a closed subspace of $\mathscr{H}(\pi)$ such that $U \pi^{\prime} U^{-1} \leqq \pi$. (Here the $U \pi^{\prime} U^{-1} \leqq \pi$ is to be interpreted concretely as stating that $U \pi^{\prime} U^{-1}$ is obtained by restricting $\pi$ to a closed invariant subspace of $\mathscr{H}(\pi)$.) Let

$$
\pi=V^{-1} \int_{B} \pi(\xi) d \nu(\xi) V
$$

be the central decomposition of $\pi$, where $B$ is a Borel subset of the quasi-dual $\tilde{G}$ and $\xi \rightarrow \pi(\xi)$ is a Borel map of $B$ into $G_{f}$ such that $\pi(\xi) \in \xi$ for all $\xi$ in $B$. Let $\pi_{1}=\int_{B} \pi(\xi) d \nu(\xi)$ and $\pi_{1}^{\prime}=V U \pi^{\prime} U^{-1} V^{-1}$. Thus $\pi_{1}^{\prime} \leqq \pi_{1}$. Let $E$ denote the projection in $\mathscr{A}\left(\pi_{1}\right)^{\prime}$ such that the space of $\pi_{1}^{\prime}$ is the range of $E$. By Corollary 2 to Proposition 4 of [3] we have

$$
\mathscr{A}\left(\pi_{1}\right)^{\prime}=\int_{B} \mathscr{A}(\pi(\xi))^{\prime} d \nu(\xi)
$$

and hence $E$ is a decomposable operator. Thus there exists a $\nu$-measurable field of projections $\xi \rightarrow E(\xi)$ on $B$ such that $E(\xi) \in \mathscr{A}(\pi(\xi))^{\prime}$ for $\nu$-almost all $\xi$. For each $\xi$ for which $E(\xi) \neq 0$, let $\pi_{1}^{\prime}(\xi)$ denote the restriction of $\pi(\xi)$ to the range of $E(\xi)$. By eliminating a set of measure zero if necessary, we may assume that $\xi \rightarrow E(\xi)$ is in fact a Borel field of projections, on $B$. Then $\{\xi: \xi \in B$ and $E(\xi)=0\}$ is a Borel subset of $B$. Thus there exists a Borel subset $B^{\prime}$ of $B$ such that $E(\xi) \neq 0$ if $\xi \in B^{\prime}$ and $E(\xi)=0$ if $\xi \in B-B^{\prime}$. Thus $\xi \rightarrow E(\xi) \mathscr{H}(\pi(\xi))$ is a $\nu$-measurable field of Hilbert spaces on $B^{\prime}$ and $\xi \rightarrow \pi_{1}^{\prime}(\xi)$ is a $\nu$-measurable field of representations on $B^{\prime}$ and

$$
\pi_{1}^{\prime}=\int_{B^{\prime}} \pi_{1}^{\prime}(\xi) d \nu(\xi)
$$

We next verify that this is in fact a central decomposition of $\pi_{1}^{\prime}$. Thus we must show that the range of the associated projection valued measure is precisely the set of projections in the center of $\mathscr{A}\left(\pi_{1}^{\prime}\right)$. Let $E^{\prime}$ be a projection in the center of $\mathscr{A}\left(\pi_{1}^{\prime}\right)$. Since $\mathscr{A}\left(\pi_{1}^{\prime}\right)=\mathscr{A}\left(\pi_{1}\right)_{E}$, we know that the center of $\mathscr{A}\left(\pi_{1}^{\prime}\right)$ is $\mathscr{Z}_{E}$, where $\mathscr{Z}$ is the center of $\mathscr{A}\left(\pi_{1}\right)$ (cf. $\S 2.1$ of Chapter 1 of [1]). Thus there exists a projection $F$ in the center of $\mathscr{A}\left(\pi_{1}\right)$ such that $E^{\prime}=F_{E}$. Since $\pi_{1}=\int_{B} \pi(\xi) d \nu(\xi)$ is the central decomposition, there exists a Borel set $B_{F} \subset B$ such that $F$ is the projection of $\mathscr{H}\left(\pi_{1}\right)$ onto $\int_{B_{F}} \mathscr{H}(\pi(\xi)) d \nu(\xi)$. Let $B_{E^{\prime}}=B_{F} \cap B^{\prime}$. Then $E^{\prime}$ is precisely the projection of $\mathscr{H}\left(\pi_{1}^{\prime}\right)$ onto $\int_{B_{E^{\prime}}} \pi_{1}^{\prime}(\xi) d \nu(\xi)$. Thus $E^{\prime}$ is contained. in the range of the projection valued measure associated with the decomposition $\pi_{1}^{\prime}=\int_{B^{\prime}} \pi_{1}^{\prime}(\xi) d \nu(\xi)$. 
Conversely, if $E^{\prime}$ is in the range of the projection valued measure, then there is a Borel set, say $B_{E^{\prime}} \subset B^{\prime}$ such that $E^{\prime}$ is the projection of $\mathscr{H}\left(\pi_{1}^{\prime}\right)$ onto $\int_{B_{E^{\prime}}} \mathscr{H}\left(\pi_{1}^{\prime}(\xi)\right) d \nu(\xi)$. Further $B_{E^{\prime}}$ is also a Borel subset of $B$. Hence there is a projection $F$ in the center of $\mathscr{A}\left(\pi_{1}\right)$, with range space $\int_{B_{E^{\prime}}} \mathscr{H}\left(\pi_{1}(\xi)\right) d \nu(\xi)$. Thus $E^{\prime}=F_{E}$ is in the center of $\mathscr{A}\left(\pi_{1}^{\prime}\right)$, since the center of $\mathscr{A}\left(\pi_{1}^{\prime}\right)$ is exactly $\mathscr{Z}_{E}$, where $\mathscr{Z}$ is the center of $\mathscr{A}\left(\pi_{1}\right)$. Thus we have established that $\pi_{1}^{\prime}=\int_{B^{\prime}} \pi_{1}^{\prime}(\xi) d \nu(\xi)$ is the central decomposition.

Next consider another central decomposition of $\pi_{1}^{\prime}$, for which all the component representations are elements of our concrete dual $G^{c}$. Thus consider the central decomposition

$$
\pi_{1}^{\prime}=W^{-1} \int_{B^{\prime}} \pi_{2}^{\prime}(\xi) d \nu(\xi) W
$$

where $\xi \rightarrow \pi_{2}^{\prime}(\xi)$ is a Borel map of $B^{\prime}$ into $G_{f}$ and $W$ is a linear isometry of $\mathscr{H}\left(\pi_{1}^{\prime}\right)$ onto $\int_{B^{\prime}} \mathscr{H}\left(\pi_{2}^{\prime}(\xi)\right) d \nu(\xi)$. Since we have two central decompositions of $\pi_{1}^{\prime}$, we may use Proposition 8.2.4 of [2] to conclude that there exists a $\nu$-measurable field of isometries $\xi \rightarrow W(\xi)$ on $B^{\prime}$ such that

$$
\pi_{1}^{\prime}(\xi)=W(\xi)^{-1} \pi_{2}^{\prime}(\xi) W(\xi)
$$

$\nu$-almost everywhere.

We are finally in a position to apply axiom (iii) of Definition 2.2. For each $\xi \in B^{\prime}, \pi_{2}^{\prime}(\xi) \in G_{f}$ and $\pi(\xi) \in G_{f}$, and further

$$
W(\xi)^{-1} \pi_{2}^{\prime}(\xi) W(\xi)=\pi_{1}^{\prime}(\xi) \leqq \pi(\xi)
$$

for $\nu$-almost all $\xi$.

Thus, for $\nu$-almost all $\xi, \mathscr{H}\left(W(\xi)^{-1} \pi_{2}^{\prime}(\xi) W(\xi)\right)$ is a closed invariant subspace for the operator $T(\pi(\xi))$ and the restriction of $T(\pi(\xi))$ to this invariant subspace is precisely $W(\xi)^{-1} T\left(\pi_{2}^{\prime}(\xi)\right) W(\xi)$. Hence the restriction of $\int_{B} T(\pi(\xi)) d \nu$ to $\int_{B^{\prime}} \mathscr{H}\left(\pi_{1}^{\prime}(\xi)\right) d \nu(\xi)$ is

$$
\int_{B^{\prime}} W(\xi)^{-1} T\left(\pi_{2}^{\prime}(\xi)\right) W(\xi) d \nu(\xi)=W^{-1} \int_{B^{\prime}} T\left(\pi_{2}^{\prime}(\xi)\right) d \nu(\xi) W .
$$

Hence the restriction of $T^{c}(\pi)=V^{-1} \int_{B} T(\pi(\xi)) d \nu(\xi) V$ to $V^{-1} \int_{B^{\prime}} \mathscr{H}\left(\pi_{1}^{\prime}(\xi)\right) d \nu(\xi) V$ $=V^{-1} \mathscr{H}\left(\pi_{1}^{\prime}\right) V=\mathscr{H}\left(U \pi^{\prime} U^{-1}\right)$ is

$$
V^{-1} W^{-1} \int_{B^{\prime}} T\left(\pi^{\prime}(\xi)\right) d \nu(\xi) W V
$$

Note that

$$
\pi^{\prime}=U^{-1} V^{-1} \pi_{1}^{\prime} V U=U^{-1} V^{-1} W^{-1} \int_{B^{\prime}} \pi_{2}^{\prime}(\xi) d \nu(\xi) W V U
$$

is a central decomposition of $\pi^{\prime}$. Hence by our definition of the extension $T^{c}$ we have

$$
T^{c}\left(\pi^{\prime}\right)=U^{-1} V^{-1} W^{-1} \int_{B^{\prime}} T\left(\pi_{2}^{\prime}(\xi)\right) d \nu(\xi) W V U
$$


Thus $U T^{c}\left(\pi^{\prime}\right) U^{-1}$ is precisely the restriction of $T^{c}(\pi)$ to $\mathscr{H}\left(U \pi^{\prime} U^{-1}\right)$ and we have shown that $T^{c}$ satisfies axiom (iii) for an option on $G^{c}$.

REMARK 2.8. Now that we have established that $T^{c}$ is an option on $G^{c}$, we shall assume the obvious extension of $T^{c}$ to any other separable representation of $G$. Indeed if $\pi$ is any such separable representation then it is unitary equivalent to some element of $G^{c}$, say $\pi=U \pi^{\prime} U^{-1}$, where $U$ is a linear isometry and $\pi^{\prime} \in G^{c}$. One then defines $T^{c}(\pi)=U T^{c}\left(\pi^{\prime}\right) U^{-1}$. The fact that $T^{c}$ satisfies axiom (iii) on $G^{c}$ ensures that $T^{c}(\pi)$ is well defined. The purpose of this convention, which after the previous proof the reader can appreciate, is to avoid the proliferation of linear isometrics.

Definition 2.9. Let $\pi=\int_{S} \pi(\xi) d \nu(\xi)$ be any direct integral decomposition of $\pi$, where $\nu$ is a $\sigma$-finite Borel measure on the standard Borel space $S$ and $\xi \rightarrow \pi(\xi)$ is a $\nu$-measurable field of representations. Such a decomposition will be said to be coarse if any one of the following equivalent conditions hold.

(i) The range of the associated projection valued measure is contained in the center of $\mathscr{A}(\pi)$.

(ii) $\mathscr{A}(\pi)=\int_{S} \mathscr{A}(\pi(\xi)) d \nu(\xi)$.

(iii) $\mathscr{A}(\pi)^{\prime}=\int_{S} \mathscr{A}(\pi(\xi))^{\prime} d \nu(\xi)$.

We shall say such a decomposition is fine if it is a decomposition into irreducible components, which is equivalent to saying that the range of the associated projection valued measure is a maximal Boolean algebra of projections in the commutant of the representation.

The equivalence of conditions (i), (ii) and (iii) is shown in Proposition 4 and Corollary 2 of [3]. The only decompositions which happen to be both coarse and fine are the central decompositions of multiplicity free representations. (A representation is multiplicity free if it has an abelian commutant.) Furthermore every decomposition may be further refined to obtain a fine decomposition (cf. Lemma 1.9 of [10]). Our next proposition states that Borel options behave well relative to coarse decompositions.

Proposition 2.10. Let $T$ be a Borel option on $G_{f}$ and let $T^{c}$ denote its canonical extension to $G^{c}$. If $\pi=\int_{S} \pi(\lambda) d \nu(\lambda)$ is a coarse decomposition, then

$$
T^{c}(\pi)=\int_{S} T^{c}(\pi(\lambda)) d \nu(\lambda)
$$

Proof. We shall prove this assertion by using the fact that every coarse decomposition may be further refined into the central decomposition (cf. Theorem 2.11 of [5] and p. 100 of [6]). More explicitly, each $\pi(\lambda)$, for $\lambda$ in $S$, admits a central decomposition into factor representations, say

$$
\pi(\lambda)=\int_{S_{\lambda}} \pi(\lambda, \gamma) d \mu_{\lambda}(\gamma)
$$


Then the double integral

$$
\pi=\int_{S} \int_{S_{\lambda}} \pi(\lambda, \gamma) d \mu_{\lambda}(\gamma) d \nu(\lambda)
$$

is equivalent to the central decomposition of $\pi$. But by its definition, the extended option $T^{c}$ integrates properly with respect to the central decomposition. Applying this fact to the central decomposition above we have

$$
T^{c}(\pi)=\int_{S} \int_{S_{\lambda}} T^{c}(\pi(\lambda, \gamma)) d \mu_{\lambda}(\gamma) d \nu(\lambda)
$$

and

$$
T^{c}(\pi(\lambda))=\int_{S_{\lambda}} T^{c}(\pi(\lambda, \gamma)) d \mu_{\lambda}(\gamma)
$$

for each $\lambda$ is $S$.

Substituting the second equation in the first we have

$$
T^{c}(\pi)=\int_{S} T^{c}(\pi(\lambda)) d \nu(\lambda)
$$

We have seen that any option on $G^{c}$ which is of the form $T^{c}$, for some Borel option $T$ on $G_{f}$, is completely determined by its values on the factor dual $G_{f}$. We define an option $T$ on $G^{c}$ to be factorizable if, whenever $\pi=\int_{\tilde{G}} \pi(\lambda) d \nu(\lambda)$ is a central decomposition of $\pi$ into factor representations, one has

$$
T(\pi)=\int_{\tilde{G}} T(\pi(\lambda)) d \nu(\lambda)
$$

Many, but certainly not all, options on $G^{c}$ are factorizable. In our remarks following Definition 2.6 we gave an example of an option on $G^{c}$ (a projection) which was not factorizable.

Proposition 2.11. The set $\mathscr{F}$ of factorizable options on $G^{c}$ is a $C^{*}$-subalgebra of the enveloping von Neumann algebra $\mathscr{A}(G)$, which contains both $G$ and its $C^{*}$-group algebra $C^{*}(G)$ and which is closed under the operation of taking strong sequential limits.

We delay the proof of Proposition 2.11 to make some remarks. The characterization of the enveloping von Neumann algebra $\mathscr{A}(G)$, as a *-algebra of options on $G^{c}$ is described in [4]. The locally compact group $G$ and its $C^{*}$-group algebra are isomorphically embedded in $\mathscr{A}(G)$. The corresponding proposition for $C^{*}$-algebras would state that the factorizable options form a $C^{*}$-subalgebra of the enveloping von Neumann algebra $\mathscr{A}(A)$ of the $C^{*}$-algebra $A$, which contains $A$ and is closed under strong sequential limits.

Since $C^{*}(G)$, when embedded in $\mathscr{A}(G)$, is strongly dense in $\mathscr{A}(G)$, it is clear that the set of factorizable options are also strongly dense in $\mathscr{A}(G)$. Nevertheless, even 
though $G$ is separable, the enveloping von Neumann algebra is so large that $\mathscr{A}(G)$ is generally not of countable type and the strong operator topology of $\mathscr{A}(G)$ does not satisfy the first axiom of countability. Thus there are many elements of $\mathscr{A}(G)$ which cannot be reached by strongly convergent sequences of factorizable options. It would be very interesting to find some other characterizations of this $C^{*}$ subalgebra of $\mathscr{A}(G)$.

Proof of Proposition 2.11. It is clear that the options corresponding to elements of $G$ or $C^{*}(G)$ are factorizable. We next observe that the factorizable options form $a^{*}$-algebra. Let $T_{1}, T_{2} \in \mathscr{F}$ and let $\alpha, \beta$ be complex numbers. Let

$$
\pi=\int_{S} \pi(\lambda) d \nu(\lambda)
$$

be a central decomposition. Then by Proposition 4 of [3],

$$
\mathscr{A}(\pi)=\int_{S} \mathscr{A}(\pi(\lambda)) d \nu(\lambda)
$$

Further $T_{1}(\pi)$ and $T_{2}(\pi)$ are elements of $\mathscr{A}(\pi)$. Since $T_{1}$ and $T_{2}$ are factorizable we have

$$
T_{i}(\pi)=\int_{S} T_{i}(\pi(\lambda)) d \nu(\lambda), \quad i=1,2
$$

By Proposition 3, p. 159 of [1], we have

$$
\begin{aligned}
\left(\alpha T_{1}+\beta T_{2}\right)(\pi) & =\alpha T_{1}(\pi)+\beta T_{2}(\pi) \\
& =\alpha \int_{S} T_{1}(\pi(\lambda)) d \nu(\lambda)+\beta \int_{S} T_{2}(\pi(\lambda)) d \nu(\lambda) \\
& =\int_{S}\left[\alpha T_{1}(\pi(\lambda))+\beta T_{2}(\pi(\lambda))\right] d \nu(\lambda) \\
& =\int_{S}\left(\alpha T_{1}+\beta T_{2}\right)(\pi(\lambda)) d \nu(\lambda) .
\end{aligned}
$$

Similarly Proposition 3, p. 159 of [1] implies that $T_{1} T_{2}$ and $T_{1}^{*}$ are factorizable. Thus $\mathscr{F}$ is a *algebra.

Next suppose $\left\{T_{i}\right\}$ is a sequence in $\mathscr{F}$ and $\left\{T_{i}\right\}$ converges strongly to $T$ in $\mathscr{A}(G)$. Then $\left\{T_{i}(\pi)\right\}$ converges strongly to $T(\pi)$. Since $T(\pi) \in \mathscr{A}(\pi)$ and $\mathscr{A}(\pi)$ $=\int_{S} \mathscr{A}(\pi(\lambda)) d \nu(\lambda)$ there exists a $\nu$-measurable field of operators $\lambda \rightarrow T(\lambda)$ such that $T(\lambda) \in \mathscr{A}(\pi(\lambda))$ for $\nu$-almost all $\lambda$ and $T(\pi)=\int_{S} T(\lambda) d \nu(\lambda)$. Since $T_{i}(\pi)$ converges strongly to $T(\pi)$, Proposition 4, p. 160 of [1] implies there exists a subsequence $T_{n_{k}}(\pi)$ of $T_{i}(\pi)$ such that $T_{n_{k}}(\pi(\lambda))$ converges strongly to $T(\lambda)$ for $\nu$-almost all $\lambda$. But since $T_{i} \rightarrow T$ strongly in $\mathscr{A}(G)$ we have $T_{n_{k}} \rightarrow T$ strongly in $\mathscr{A}(G)$. Thus 
$T_{n_{k}}(\pi(\lambda)) \rightarrow T(\pi(\lambda))$ strongly for all $\lambda$ in $S$. Hence we have $T(\lambda)=T(\pi(\lambda))$ for $\nu$ almost all $\lambda$ and hence

i.e., $T$ is factorizable.

$$
T(\pi)=\int_{S} T(\pi(\lambda)) d v(\lambda)
$$

It is now clear that $\mathscr{F}$ is a $C^{*}$-algebra. If $T$ is contained in the uniform closure of $\mathscr{F}$, it is the uniform limit of a sequence in $\mathscr{F}$ and hence also the strong limit of this sequence in $\mathscr{F}$. By our previous result, $T$ must be contained in $\mathscr{F}$.

3. A strong duality theorem. We shall now give an application of the previous study of Borel options by obtaining a strong duality theorem for separable locally compact groups which will generalize Tatsuuma's strong form of his duality theorem (Proposition 4.1 of [10]) to the nontype I case. We will thus show how such a group may be reconstructed from its set of factor representations and its natural structure consisting of direct sums, unitary equivalence, Borel structure and tensor product structure. The direct integral theory must be used to describe the tensor product structure, as the set of factor representations is, in general, not closed with respect to the operation of tensor products. The topological part of the duality theorem, which shows how the topology of the group may be reconstituted from its factor representation theory, is new even in the type I case, and will be described in the next section.

The letter $G$, as always, will denote a separable locally compact group. In $\mathrm{N}$. Tatsuuma's strong duality theory for type I groups, he begins with the dual object $\hat{G}$ of unitary equivalence classes of irreducible representations of $G$, and then chooses a cross-section. An operator valued function (option) on this crosssection can then be extended to all concrete irreducible representations by requiring that it preserve unitary equivalence. In the nontype I case it is not surprising that we shall require a dual object consisting of factor representations rather than merely irreducible representations. Thus the quasi-dual $\tilde{G}$, the set of all quasi-equivalence classes of separable factor representations of $G$, might be a natural candidate for a dual object. However in the nontype I case, it is not possible to take a Borel cross-section of the dual $\hat{G}$, or the quasi-dual $\tilde{G}$. For this reason we shall consider the concrete factor dual $G_{f}$ (cf. Definition 2.1) as our "dual object" in this theory.

The reader may recall the definition of a Borel option on $G_{f}$ (Definitions 2.2 and 2.6). Also recall (Definition 2.9) that any direct integral decomposition of a representation into irreducible components is called a fine decomposition.

Definition 3.1. An option $T$ on $G_{f}$ is said to be admissible if the following three conditions are satisfied:

(i) $T$ is a Borel option on $G_{f}$.

(ii) $T(\pi) \neq 0$ for every $\pi$ in $G_{f}$.

(iii) If $\pi_{1}, \pi_{2}$ are elements of $G_{f}$ and if

$$
U\left(\pi_{1} \otimes \pi_{2}\right) U^{-1}=\int_{S} \pi(\lambda) d \nu(\lambda)
$$


is any fine decomposition of $\pi_{1} \otimes \pi_{2}$, where $\pi(\lambda) \in G_{f}$ for all $\lambda$ in $S$, and $U$ is a linear isometry, then

$$
U\left(T\left(\pi_{1}\right) \otimes T\left(\pi_{2}\right)\right) U^{-1}=\int_{S} T(\pi(\lambda)) d \nu(\lambda) .
$$

Definition 3.2. Let $G^{+}$denote the set of all admissible options on $G_{f}$. Define multiplication pointwise; i.e., if $S, T \in G^{+}$, define $S T$ by $S T(\pi)=S(\pi) T(\pi)$ for all $\pi$ in $G_{f}$. Give $G^{+}$the largest topology such that $T_{i}$ converges to $T$ whenever $T_{i}$ is a sequence such that $T_{i}(\pi)$ converges strongly to $T(\pi)$, for all $\pi$ in $G_{f}$ (cf. Remark 4.5). We shall call $G^{+}$the reconstituted group, a terminology justified by the following theorem.

THEOREM 3.3 (THE DUALITY THEOREM IN STRONG FORM). Let $G$ be a separable locally compact group. Then the reconstituted group $G^{+}$is a separable locally compact group. To each $x$ in $G$, we may associate an option $\hat{x}$ on $G_{f}$ defined by

$$
\hat{x}(\pi)=\pi(x) \text { for all } \pi \text { in } G_{f} .
$$

The mapping $x \rightarrow \hat{x}$ is an isomorphism and homeomorphism of $G$ onto $G^{+}$.

The fact that $G^{+}$is a separable locally compact group will follow immediately when we show $x \rightarrow \hat{x}$ is an isomorphism and homeomorphism of $G$ onto $G^{+}$. The fact that $x \rightarrow \hat{x}$ is a homeomorphism will be proved in $\S 4$ when we study the topology of $G^{+}$. The map $x \rightarrow \hat{x}$ is clearly a one-to-one homomorphism. The proof that this map is surjective will depend on the following two propositions, as well as the weak form of the Tatsuuma duality theorem.

Proposition 3.4. Let $G$ be a separable locally compact group and let $T$ be an admissible option on $G_{f}$. As usual, let $T^{c}$ denote the canonical extension of $T$ to $G^{c}$ (cf. Theorem 2.7 and Remark 2.8). If

$$
U \pi U^{-1}=\int_{S} \pi(\lambda) d \nu(\lambda)
$$

is any direct integral decomposition of $\pi$, then

$$
U T^{c}(\pi) U^{-1}=\int_{S} T^{c}(\pi(\lambda)) d \nu(\lambda) .
$$

Proof. Notice that for Borel options we were able to obtain this result only for coarse decompositions. We are thus able to obtain a much stronger result for admissible options. We prove this result in three steps, by considering special cases first.

Step 1. (The case of a fine decomposition of a factor representation.) We assume $\pi$ is a factor representation of $G$ and

$$
U \pi U^{-1}=\int_{S} \pi(\lambda) d \nu(\lambda)
$$

is a fine decomposition into irreducible components. 
In axiom (iii) of Definition 3.1, let $\pi_{1}=\pi_{2}$ be the trivial one-dimensional representation $\varepsilon$ of $G$. Let $W$ denote the natural isometry of $\boldsymbol{C} \otimes \boldsymbol{C}$ onto $\boldsymbol{C}$ where $\boldsymbol{C}$ is the (one-dimensional Hilbert space of) complex numbers. Thus $W(\varepsilon \otimes \varepsilon) W^{-1}=\varepsilon$ is a (discrete) decomposition into irreducible components and hence by axiom (iii)

$$
W(T(\varepsilon) \otimes T(\varepsilon)) W^{-1}=T(\varepsilon),
$$

from which one concludes that $T(\varepsilon)^{2}=T(\varepsilon)$. Using axiom (ii) we conclude that $T(\varepsilon)=1$.

Returning to our factor representation $\pi$, there is a natural isometry $V$ of $\mathscr{H}(\pi) \otimes C$ onto $\mathscr{H}(\pi)$ defined by $V(\psi \otimes \alpha)=\alpha \psi$ for all $\alpha$ in $C$ and $\psi$ in $\mathscr{H}(\pi)$. Similarly define an isometry $V_{\lambda}$ of $\mathscr{H}(\pi(\lambda)) \otimes C$ onto $\mathscr{H}(\pi(\lambda))$. Then we have $V(\pi \otimes \varepsilon) V^{-1}=\pi$ and $V_{\lambda}(\pi(\lambda) \otimes \varepsilon) V_{\lambda}^{-1}=\pi(\lambda)$ for all $\lambda$ in $S$. Thus

$$
U V(\pi \otimes \varepsilon) V^{-1} U^{-1}=\int_{S} V_{\lambda}(\pi(\lambda) \otimes \varepsilon) V_{\lambda}^{-1} d \nu(\lambda)
$$

is a fine decomposition of $\pi \otimes \varepsilon$ and hence by axiom (iii) of Definition 3.1 we have

$$
\begin{aligned}
U V(T(\pi) \otimes T(\varepsilon)) V^{-1} U^{-1} & =\int_{S} T\left(V_{\lambda}(\pi(\lambda) \otimes \varepsilon) V_{\lambda}^{-1}\right) d \nu(\lambda) \\
& =\int_{S} T(\pi(\lambda)) d \nu(\lambda),
\end{aligned}
$$

while

$$
U V(T(\pi) \otimes T(\varepsilon)) V^{-1} U^{-1}=U V(T(\pi) \otimes 1) V^{-1} U^{-1}=U T(\pi) U^{-1} .
$$

Step 2. (The case of a fine decomposition of an arbitrary representation.) Here we assume $\pi$ is an arbitrary representation, but that

$$
U \pi U^{-1}=\int_{S} \pi(\lambda) d \nu(\lambda)
$$

is a fine decomposition.

Since the center of $\mathscr{A}(\pi)$ is contained in any maximal abelian subalgebra of $\mathscr{A}(\pi)^{\prime}$, we have that the fine decomposition $\int_{S} \pi(\lambda) d \nu(\lambda)$ is necessarily a refinement of the central decomposition of $\pi$ (cf., for example, p. 100 of [6]). Thus we may write our fine decomposition equivalently as a double integral

$$
\int_{\tilde{G}} \int_{S_{\xi}} \pi(\lambda, \xi) d \nu(\lambda) d \mu(\xi)
$$

where $\int_{\tilde{G}} \pi^{\prime}(\xi) d \mu(\xi)$ is a central decomposition and

$$
\pi^{\prime}(\xi)=\int_{S_{\xi}} \pi(\lambda, \xi) d \nu_{\xi}(\lambda)
$$

is a fine decomposition of the factor representation $\pi^{\prime}(\xi)$. Now

$$
T^{c}\left(\int_{\tilde{G}} \pi^{\prime}(\xi) d \mu(\xi)\right)=\int_{\tilde{G}} T^{c}\left(\pi^{\prime}(\xi)\right) d \mu(\xi)
$$


by Proposition 2.10. On the other hand

$$
T^{c}\left(\pi^{\prime}(\xi)\right)=\int_{S_{\xi}} T^{c}(\pi(\lambda, \xi)) d \nu_{\xi}(\lambda)
$$

by Step 1 since $\pi^{\prime}(\xi)$ is a factor representation. Thus

$$
T^{c}\left[\int_{\tilde{G}} \int_{S_{\xi}} \pi(\lambda, \xi) d \nu_{\xi}(\lambda) d \mu(\xi)\right]=\int_{\tilde{G}} \int_{S_{\xi}} T^{c}(\pi(\lambda, \xi)) d \nu_{\xi}(\lambda) d \mu(\xi) .
$$

Putting this in the equivalent single integral form we have

$$
T^{c}\left[\int_{S} \pi(\lambda) d \nu(\lambda)\right]=\int_{S} T^{c}(\pi(\lambda)) d \nu(\lambda)
$$

Thus (cf. Remark 2.8) we have

$$
\begin{aligned}
U T^{c}(\pi) U^{-1} & =T^{c}\left(U \pi U^{-1}\right) \\
& =T^{c}\left(\int_{S} \pi(\lambda) d \nu(\lambda)\right) \\
& =\int_{S} T^{c}(\pi(\lambda)) d \nu(\lambda) .
\end{aligned}
$$

Step 3. (The general case.) A general decomposition $\int_{S} \pi(\lambda) d \nu(\lambda)$ admits a refinement into a fine decomposition (cf. Lemma 1.9 of [10]). Thus each $\pi(\lambda)$ admits a fine decomposition

$$
\pi(\lambda)=\int_{s_{\lambda}} \pi(\lambda, \xi) d \nu_{\lambda}(\xi)
$$

so that

$$
U \pi U^{-1}=\int_{S} \int_{S_{\lambda}} \pi(\lambda, \xi) d \nu_{\lambda}(\xi) d \nu(\lambda)
$$

is also a fine decomposition. By Step 2 we have

$$
T^{c}(\pi(\lambda))=\int_{S_{\lambda}} T^{c}(\pi(\lambda, \xi)) d \nu_{\lambda}(\xi)
$$

and again by Step 2

$$
U T^{c}(\pi) U^{-1}=\int_{S} \int_{S_{\lambda}} T^{c}(\pi(\lambda, \xi)) d \nu_{\lambda}(\xi) d \nu(\lambda) .
$$

Hence

$$
U T^{c}(\pi) U^{-1}=\int_{S} T^{c}(\pi(\lambda)) d \nu(\lambda) .
$$

COROllary 3.5. If $T$ is an admissible option on $G_{f}$, then

$$
T^{c}\left(\pi_{1} \otimes \pi_{2}\right)=T\left(\pi_{1}\right) \otimes T\left(\pi_{2}\right)
$$

for any $\pi_{1}, \pi_{2}$ in $G_{f}$. 
Proof. According to axiom (iii) of Definition 3.1, if

$$
U\left(\pi_{1} \otimes \pi_{2}\right) U^{-1}=\int_{S} \pi(\lambda) d \nu(\lambda)
$$

is a fine decomposition (and every separable representation admits a fine decomposition) then

$$
T\left(\pi_{1}\right) \otimes T\left(\pi_{2}\right)=U^{-1} \int_{S} T(\pi(\lambda)) d \nu(\lambda) U
$$

By Proposition 3.4 we have

$$
U^{-1} \int_{S} T(\pi(\lambda)) d \nu(\lambda) U=U^{-1} T^{c}\left[\int_{S} \pi(\lambda) d \nu(\lambda)\right] U=T^{c}\left(\pi_{1} \otimes \pi_{2}\right)
$$

Our next proposition generalizes this property to representations which are not factor representations.

Proposition 3.6. If $T$ is an admissible option on $G_{f}$ and $\pi_{1}$ and $\pi_{2}$ are any two separable representations of $G$, then

$$
T^{c}\left(\pi_{1} \otimes \pi_{2}\right)=T^{c}\left(\pi_{1}\right) \otimes T^{c}\left(\pi_{2}\right) .
$$

Proof. Suppose first that $\pi_{1}$ is a factor representation. Let

$$
\pi_{2}=V^{-1} \int_{S} \pi_{2}(\lambda) d \nu(\lambda) V
$$

be a central decomposition of $\pi_{2}$. Then there exists a linear isometry $U$ such that

$$
\pi_{1} \otimes \pi_{2}=U^{-1} \int_{S}\left(\pi_{1} \otimes \pi_{2}(\lambda)\right) d \nu(\lambda) U
$$

(This is a special case of Lemma 1.10 of [10], and also follows from Proposition 11, p. 152 of [1].) Since $\pi_{1}$ and $\pi_{2}(\lambda)$ are factor representations, Corollary 3.5 implies

$$
T^{c}\left(\pi_{1} \otimes \pi_{2}(\lambda)\right)=T^{c}\left(\pi_{1}\right) \otimes T^{c}\left(\pi_{2}(\lambda)\right) .
$$

On the other hand, by Proposition 3.4 we have

$$
\begin{aligned}
T^{c}\left(\pi_{1} \otimes \pi_{2}\right) & =U^{-1} \int_{S} T^{c}\left(\pi_{1} \otimes \pi_{2}(\lambda)\right) d \nu(\lambda) U \\
& =U^{-1} \int_{S} T^{c}\left(\pi_{1}\right) \otimes T^{c}\left(\pi_{2}(\lambda)\right) d \nu(\lambda) U \\
& =T^{c}\left(\pi_{1}\right) \otimes V^{-1} \int_{S} T^{c}\left(\pi_{2}(\lambda)\right) d \nu(\lambda) V \\
& =T^{c}\left(\pi_{1}\right) \otimes T^{c}\left[V^{-1} \int_{S} \pi_{2}(\lambda) d \nu(\lambda) V\right] \\
& =T^{c}\left(\pi_{1}\right) \otimes T^{c}\left(\pi_{2}\right) .
\end{aligned}
$$


The above argument may now be repeated in the case where $\pi_{1}$ is not a factor representation. Once again we take the central decomposition of $\pi_{2}$ and use the result just obtained to argue that

$$
T^{c}\left(\pi_{1} \otimes \pi_{2}(\lambda)\right)=T^{c}\left(\pi_{1}\right) \otimes T^{c}\left(\pi_{2}(\lambda)\right) .
$$

We conclude this section by completing the algebraic portion of the proof of the duality theorem (Theorem 3.3). It is sufficient to show the map $x \rightarrow \hat{x}$ maps $G$ onto $G^{+}$. If $T \in G^{+}$, then $T$ is an admissible option on $G_{f}$ and hence by Proposition 3.6 has a canonical extension $T^{c}$ which preserves tensor products of separable representations. If $L$ is the left regular representation, $T^{c}(L) \neq 0$ follows from axiom (ii) of Definition 3.1 by taking the central decomposition of $L$. By the Tatsuuma duality theorem (cf. Proposition 2.1 of [10] and Remark 3.14 of [4]) it follows that $T^{c}$ is of the form $T^{c}=\hat{x}$ for some $x$ in $G$, i.e., $T^{c}(\pi)=\pi(x)$ for any representation $\pi$. In particular $T(\pi)=\pi(x)$ for every $\pi$ in $G_{f}$.

4. The topology of the reconstituted group. In this section we shall consider the topological portion of the duality theorem. How can the topology of the group $G$ be reconstructed from its representation theory? It is known that the topology of $G$ is precisely the smallest topology such that the maps $x \rightarrow \pi(x)$ are strongly continuous, for all $\pi$ in $G^{c}$ (cf. Remark 3.14 of [4]). Indeed it is known that the left regular representation $L$ of $G$ is a strong operator topology homeomorphism, and hence the topology of $G$ is the smallest topology such that $x \rightarrow L(x)$ is strongly continuous (cf. Lemma 2.2 of [4] and note that the weak and strong operator topologies are identical on the unitary group). However in the strong form of duality, we wish to regain the topology of $G$ purely from the space of factor representations. Indeed we shall see the topology of $G$ can be specified purely in terms of the irreducible representations. A simple application of the Lebesgue dominated convergence theorem enables us to reduce the problem to consideration of the left regular representation, where the situation is well understood. The crucial observation is given in the next proposition.

Proposition 4.1. Let $\pi=\int_{S} \pi(\lambda) d \nu(\lambda)$ be any direct integral decomposition, where $\pi$ is a separable representation of $G$. If $\left\{x_{i}\right\}$ is a sequence in $G$ and $x \in G$ such that $\pi\left(\lambda, x_{i}\right)$ converges strongly to $\pi(\lambda, x)$, for each $\lambda$ in $S$, then $\pi\left(x_{i}\right)$ converges strongly to $\pi(x)$.

Proof. Each $\psi \in \mathscr{H}(\pi)$ is of the form $\psi=\int_{S} \psi(\lambda) d \nu(\lambda)$ where $\lambda \rightarrow \psi(\lambda)$ is a measurable field of vectors on $S$ such that $\psi(\lambda) \in \mathscr{H}(\pi(\lambda))$ for each $\lambda$ in $S$ and

$$
\|\psi\|^{2}=\int_{s}\|\psi(\lambda)\|^{2} d \nu(\lambda)<+\infty
$$

Then

$$
\left\|\left(\pi\left(x_{i}\right)-\pi(x)\right) \psi\right\|^{2}=\int_{S}\left\|\left(\pi\left(\lambda, x_{i}\right)-\pi(\lambda, x)\right) \psi(\lambda)\right\|^{2} d \nu(\lambda)
$$


Thus

$$
f_{i}(\lambda)=\left\|\left(\pi\left(\lambda, x_{i}\right)-\pi(\lambda, x)\right) \psi(\lambda)\right\|^{2}
$$

is a positive $\nu$-measurable, and in fact $\nu$-integrable function on $S$. Further since $\pi\left(\lambda, x_{i}\right)$ converges strongly to $\pi(\lambda, x)$ for each $\lambda$ in $S$, by hypothesis, we have that $f_{i}$ converges to zero pointwise on $S$. Further each $f_{i}$ is bounded by a $\nu$-integrable function. Indeed

$$
\begin{aligned}
f_{i}(\lambda) & =\left\|\left(\pi\left(\lambda, x_{i}\right)-\pi(\lambda, x)\right) \psi(\lambda)\right\|^{2} \\
& \leqq\left\|\pi\left(\lambda, x_{i}\right) \psi(\lambda)\right\|^{2}+\|\pi(\lambda, x) \psi(\lambda)\|^{2}+2\left\|\pi\left(\lambda, x_{i}\right) \psi(\lambda)\right\|\|\pi(\lambda, x) \psi(\lambda)\| \\
& \leqq 4\|\psi(\lambda)\|^{2}
\end{aligned}
$$

where we have used the fact that $\pi$ is a unitary representation. By the Lebesgue dominated convergence theorem (cf. Corollary 32.3.3, p. 234 of [8]), we have that $f_{i}$ converges to zero in the mean. Thus

$$
\left\|\left(\pi\left(x_{i}\right)-\pi(x)\right) \psi\right\|^{2}=\int_{S}\left\|\left(\pi\left(\lambda, x_{i}\right)-\pi(\lambda, x)\right) \psi(\lambda)\right\|^{2} d \nu(\lambda)
$$

converges to zero. Hence $\pi\left(x_{i}\right)$ converges to $\pi(x)$ in the strong operator topology.

COROLlaRY 4.2. Suppose $L$ is the left regular representation of the group $G$ and suppose

$$
U L U^{-1}=\int_{S} \pi(\lambda) d \nu(\lambda)
$$

is any direct integral decomposition of $L$, where $U$ is a linear isometry. If $\left\{x_{i}\right\}$ is a sequence in $G$ and $x \in G$ such that $\pi\left(\lambda, x_{i}\right)$.converges strongly to $\pi(\lambda, x)$ for $\nu$-almost all $\lambda$ in $S$, then $x_{i}$ converges to $x$ in $G$.

Proof. By Proposition 4.1, $L\left(x_{i}\right)$ converges strongly to $L(x)$. But (Lemma 2.2 of [4]) $L$ is a strong operator topology homeomorphism.

COROLlary 4.3. Let $x_{i}$ be a sequence in $G$ and $x \in G$. Then $x_{i}$ converges to $x$ if and only if $\pi\left(x_{i}\right)$ converges strongly to $\pi(x)$, for every $\pi$ in $G_{f}$.

Proof. Every $\pi$ in $G_{f}$ is strongly continuous. On the other hand we may take the central decomposition of the left regular representation $L$, in Corollary 4.2 , so that all the components $\pi(\lambda)$ are factor representations in $G_{f}$.

COROLlaRY 4.4. The map $x \rightarrow \hat{x}$ of $G$ onto the reconstituted group $G^{+}$given in Theorem 3.3 is a homeomorphism.

Proof. This is simply a reformulation of Corollary 4.3. This corollary completes the proof of Theorem 3.3.

COROllary 4.5. A sequence $\left\{x_{i}\right\}$ in $G$ converges to an element $x$ in $G$ if and only if $\pi\left(x_{i}\right)$ converges strongly to $\pi(x)$, for every irreducible representation of $G$. 
Proof. By irreducible representation we always mean strongly continuous unitary irreducible representation. On the other hand we may consider any fine decomposition of the left regular representation, in Corollary 4.2, so that all the components are irreducible.

REMARK 4.6. We are indebted to Charles Akemann for discussions on which this remark is based. In particular he suggested the example described below.

We have defined the topology on $G^{+}$as the largest such that $T_{i}$ converges to $T$ whenever $T_{i}$ is a sequence such that $T_{i}(\pi)$ converges strongly to $T(\pi)$, for all $\pi$ in $G_{f}$. This topology may be described more explicitly by saying a set $F$ in $G^{+}$is closed if $F$ contains $T$, whenever $T_{i}$ is a sequence in $F$ such that $T_{i}(\pi)$ converges strongly to $T(\pi)$ for all $\pi$ in $G_{f}$. One may easily verify that this collection of sets is closed under finite unions and arbitrary intersections, and thus determines a topology for $G^{+}$. Further it is easy to see that this is the largest topology on $G^{+}$such that $T_{i}$ converges to $T$ whenever $T_{i}$ is a sequence such that $T_{i}(\pi)$ converges strongly to $T(\pi)$ for all $\pi$ in $G_{f}$.

Proposition 4.1 implies the topology of $G$, and hence of $G^{+}$, may be characterized in a completely similar manner, using certain subsets $S$ of $G_{f}$. Thus the topology of $G^{+}$is equivalently defined using the set of irreducible representations in $G_{f}$ rather than the entire set $G_{f}$. Moreover the set of irreducible representations appearing in any fine decomposition of the left regular representation will do just as well.

The characterization of the topology of $G^{+}$in terms of sequential convergence rather than in terms of nets is crucial. Thus the hypothesis that $G$ is first countable is essential, in order that its topology be characterized in terms of sequential convergence. More explicitly, Corollaries 4.3 and 4.5 are false if one uses nets instead of sequences. Even the additive real line $\boldsymbol{R}$ is a counterexample for the corresponding statements for nets. Indeed let $\boldsymbol{R}_{B}$ denote the Bohr compactification of $\boldsymbol{R}$. Then the sequence of positive integers in $\boldsymbol{R}$ admits a subnet $\left\{N_{\lambda}: \lambda \in \Lambda\right\}$ which converges, in $\boldsymbol{R}_{B}$, to some element in $\boldsymbol{R}_{B}$. Thus the net

$$
\left\{N_{\lambda}-N_{\lambda^{\prime}}:\left(\lambda, \lambda^{\prime}\right) \in \Lambda \times \Lambda\right\}
$$

is a net in $\boldsymbol{R}$ which converges to 0 , relative to the topology of $\boldsymbol{R}_{B}$. Clearly this net does not converge to 0 in the usual topology of $\boldsymbol{R}$. However, by definition of the topology of the Bohr compactification, the net $\left\{\varphi\left(N_{\lambda}-N_{\lambda^{\prime}}\right):\left(\lambda, \lambda^{\prime}\right) \in \Lambda \times \Lambda\right\}$ converges to zero, for every continuous character $\varphi$ of $\boldsymbol{R}$, i.e., for every irreducible representation of $\boldsymbol{R}$.

\section{REFERENCES}

1. J. Dixmier, Les algèbres d'opérateurs dans l'espace Hilbertien, 2nd ed., Gauthier-Villars, Paris, 1969.

2. - Les $C^{*}$-algebres et leurs représentations, Cahiers Scientifiques, fasc. 29, GauthierVillars, Paris, 1964. MR 30 \#1404.

3. J. A. Ernest, A decomposition theory for unitary representations of locally compact groups, Trans. Amer. Math. Soc. 104 (1962), 252-277. MR 25 \#3383. 
4. J. A. Ernest, Hopf-von Neumann algebras, Proc. Conf. Functional Analysis (University of California, Irvine, Calif., 1966), Academic Press, London; Thompson, Washington, D. C., 1967, pp. 195-215. MR 36 \#6956.

5. G. W. Mackey, Induced representations of locally compact groups. II; The Frobenius reciprocity theorem, Ann. of Math. (2) 58 (1953), 193-221. MR 15, 101.

6. — The theory of group representations, University of Chicago, Chicago, Ill., 1955 (mimeographed notes).

7. - Borel structure in groups and their duals, Trans. Amer. Math. Soc. 85 (1957), 134165. MR 19, 752.

8. M. E. Munroe, Introduction to measure and integration, Addison-Wesley, Reading, Mass., 1953. MR 14, 734.

9. M. Takesaki, $A$ duality in the representation theory of $C^{*}$-algebras, Ann. of Math. (2) 85 (1967), 370-382. MR 35 \#755.

10. N. Tatsuuma, $A$ duality theorem for locally compact groups, J. Math. Kyoto Univ. 6 (1967), 187-293. MR 36 \#313.

\section{UNIVERSity OF CALIFoRNia, \\ Santa Barbara, California 93106}

